\title{
Effect of Tirofiban on new cerebral microbleeds after mechanical thrombectomy in patients with acute ischemic stroke
}

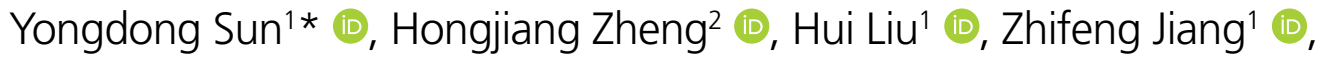 \\ Yongying Ren ${ }^{1} \mathbb{D}^{\mathbb{D}}$, Xin Huang ${ }^{1} \mathbb{C}^{\circ}$, Lihua Wang ${ }^{1}$
}

\begin{abstract}
SUMMARY
OBJECTIVE: The aim of this study was to analyze the effect of tirofiban on new cerebral microhemorrhage after mechanical thrombectomy in patients with acute ischemic stroke.

METHODS: In total, 203 patients with acute ischemic stroke treated by mechanical thrombectomy in our department of neurology were enrolled as the research objects. The patients were divided into two groups: the patients who used tirofiban within $24 \mathrm{~h}$ after surgery were assigned to the study group (78 subjects), while patients who did not use tirofiban were assigned to the conventional group (125 subjects). Magnetic resonance imaging was used to detect new-onset cerebral microbleeds in patients with stroke after surgery. The National Institute of Health Stroke Scale, modified ranking scale, and activity of daily living scale were used to assess the prognosis of patients, and the general data and the occurrence of adverse effects between two groups were compared to comprehensively evaluate the efficacy and safety of tirofiban.

RESULTS: The proportion of atrial fibrillation in the research group was significantly lower than that in the conventional group. The research group had a much lower rate of new-onset cerebral microbleeds than the conventional group $(p<0.001)$. There was no significant difference in the proportion of adverse reactions between the two groups ( $p>0.05)$.

CONCLUSION: The application of tirofiban in mechanical thrombectomy of patients with acute ischemic stroke has high safety, effectively reduces the occurrence of new cerebral microhemorrhage, and provides a guarantee for patient safety.

KEYWORDS: Tirofiban. Acute ischemic stroke. Neurologic deficits.
\end{abstract}

\section{INTRODUCTION}

Acute cerebral infarction often leads to neurological impairment in patients. It is one of the common critical illnesses in the emergency department of hospitals and has a high incidence $^{1,2}$. Intravascular mechanical thrombectomy is widely used in clinical practice due to the gradual extension of the "time window" and higher opening rate. However, due to mechanical damage to the vascular endothelium, etc., platelet activation will be formed after thrombosis occurs. One of the important links of mechanical thrombectomy treatment is how to quickly and effectively inhibit excessive platelet activation for such patients ${ }^{3}$. To reduce the risk of occlusion, tirofiban can be injected intravenously during and after the operation. It is reported that tirofiban, a highly selective, fast-acting non-peptide glycoprotein IIb/IIIa (Gp IIb/IIIa) platelet receptor antagonist, could reversibly block the specific binding of platelets

\footnotetext{
1Jizhong Energy Fengfeng Group General Hospital, Department of Neurosurgery - Handan, China.

${ }^{2}$ Jizhong Energy Fengfeng Group General Hospital, Department of Pathology - Handan, China.

*Corresponding author: sunyongdon@163.com

Conflicts of interest: the authors declare there are no conflicts of interest. Funding: none.

Received on August 18, 2021. Accepted on August 19, 2021.
} 
to fibrinogen ligands and directly inhibit platelet aggregation. Tirofiban is not immunogenic, so it also does not activate complement to damage platelets. The antiplatelet effects would readily be apparent after five min of intravenous infusion, and the bleeding time would normalize $4 \mathrm{~h}$ after discontinuation ${ }^{4,5}$.

A tiny intracerebral hemorrhage that cannot be detected by conventional computed tomography (CT) is called cerebral microbleeds (CMBs). It will occur in the acute phase of patients with acute ischemic stroke, and the incidence rate is higher than symptomatic intracranial hemorrhage. Studies have confirmed that patients with ischemic stroke with new-onset CMBs are more susceptible to symptomatic intracranial hemorrhage, which indicate that CMBs may be one of the risk factors for symptomatic intracranial hemorrhage ${ }^{6}$. Researches ${ }^{7-9}$ have shown that the use of tirofiban in patients with acute ischemic stroke does not increase the risk index of symptomatic intracranial hemorrhage in the acute phase. However, a few studies have been carried out to clarify the specific application safety of tirofiban on CMBs in patients with acute ischemic stroke. Therefore, we conducted a prospective case-control study to analyze the impact of tirofiban on new CMBs after mechanical thrombectomy in patients with acute ischemic stroke and related risk factors.

\section{METHODS}

\section{General information}

In total, 203 patients with acute ischemic stroke treated in the Department of Neurology in our hospital from January 2017 to December 2020 were enrolled in this study as the research objects. The patients were grouped according to the use of tirofiban within $24 \mathrm{~h}$ after operation. The patients who used tirofiban and those who did not use tirofiban were divided into the research group and the conventional group, respectively. The number of patients in the above two groups was 78 and 125 , respectively.

The inclusion criteria were as follows:

(1) age older than 16 years;

(2) patients with acute ischemic stroke were treated with mechanical thrombectomy;

(3) occlusion of the intracranial segment of the internal carotid artery, the M1 or M2 segment of the middle cerebral artery, and the front A1 segment of the brain to cause infarction;

(4) the National Institute of Health Stroke Scale (NIHSS) score was 6 points or above;

(5) the Diffusion-Weighted Imaging-Alberta Stroke Program Early Computed Tomography Score (DWI-ASPECT) $\geq 6$ points; and
(6) informed consent signed by patients or their agents.

The exclusion criteria were as follows:

(1) the modified rankin scale ( $m R S$ ) $\geq 3$ points before the onset of the disease and

(2) head CT detection showed changes in cerebral hemorrhage or early cerebral infarction with low density or higher than one-third of one hemisphere and other obvious early cerebral infarction changes.

\section{Mechanical thrombectomy method}

The specific operation of the mechanical thrombectomy method is as follows. The whole cerebral angiography is performed on the patient. If the result of the angiography shows that there is no recanalization of the responsible artery, the micro-guide wire and micro-catheter are passed through the guide catheter to the distal end of the thrombus. The Solitaire ${ }^{\mathrm{Tm}}$ FR blood flow reconstruction device produced by Medtronic, Minneapolis, MN, USA is used to repeatedly deliver to the distal end of the thrombus to open and retract it to the outside of the body to remove the whole or divided thrombus, and the above whole process can be repeated. After the operation is completed, the opening of the occluded artery is confirmed. The above operation is repeated until the occluded artery is opened, but the number of repetitions should be controlled within five times. During the treatment activities, the patient's vital signs, consciousness, limb activities, etc., should be closely monitored. If bleeding occurs, the operation must be stopped immediately. Attention should be paid to the patients in order to reduce the adverse reactions after the operation. The enrolled cases are admitted to the stroke unit, and those with the severe illness must be admitted to the intensive care unit, and all patients will undergo rehabilitation training after their illness is stable ${ }^{7,8}$.

\section{Use of medication}

If there is a high risk of reocclusion such as permanent stent implantation and large vessel stenosis during the treatment, the treatment will start during the operation. The specification of tirofiban (National Medicine Standard: H20041165) manufactured by the Chinese pharmaceutical company Yuanda Pharmaceutical is $100 \mathrm{ml}$. Later, it is changed to a maintenance dose of $0.12 \mathrm{~kg}^{-1} \cdot \mathrm{h}^{-1}$ for $24 \mathrm{~h}$ (if there is no bleeding symptom during the 18 -h reexamination, then give aspirin $300 \mathrm{mg}$ and clopidogrel $75 \mathrm{mg}$, and bridging with tirofiban for $6 \mathrm{~h}$ ). For patients in the conventional group who did not use tirofiban during the treatment process, they were treated with oral antiplatelets (aspirin/clopidogrel) when there was no bleeding on the head CT after $24 \mathrm{~h}$ (aspirin $100 \mathrm{mg}$ and clopidogrel 75 $\mathrm{mg}$ once a day $)^{9,10}$. 


\section{Magnetic resonance imaging examination of cerebral microbleeds}

Notably, $24 \mathrm{~h}$ after completing the above operations, the patients are reexamined with CT or MRI. All patients in the group are examined on the head susceptibility-weighted imaging (SWI) within $48 \mathrm{~h}$ after the onset of the disease and 8 days after admission. SWI usually can detect minor bleeding after $48 \mathrm{~h}$. Therefore, SWI examination within $48 \mathrm{~h}$ of onset can confirm old CMBs, but SWI examination at about eight days after onset can observe the condition of new CMBs. Four imaging specialists read the SWI images and analyze whether they are new images of microbleeds ${ }^{11}$.

\section{Observation indicators}

\section{General information}

General information, such as age, sex, history of smoking, history of drinking, history of atrial fibrillation routine blood, urine routine, blood lipids, fasting blood glucose, and other laboratory test indicators of the patients after admission, were collected and retrospectively analyzed.

\section{Clinical indicators}

(1) Use NIHSS to assess the patient's stroke status before and 7, 14, and 28 days after treatment. The lower the score indicated the less severe the condition.

(2) Use mRS to evaluate the prognosis of the enrolled patients on 7, 14, and 28 days after treatment. The lower the score indicated the better the prognosis.

(3) The activity of daily living (ADL) scale (Barthel Index) was assessed on 7, 14, and 28 days after treatment and divided into two categories: satisfactory (Barthel Index $\geq 95$ points) and dissatisfaction (Barthel Index $<95$ points) index.

\section{Safety indicators}

To study the occurrence of adverse effects, the number of bleeding events three months after discharge should be followed up, according to the bleeding grading standard of the reported infarction-related artery-opening strategy:

(1) severe life-threatening bleeding;

(2) if there is moderate bleeding, blood transfusion is needed; and

(3) slight bleeding such as bleeding gums, ecchymoses under the skin, etc., and whether there are any allergic reactions.

\section{Statistical methods}

In this study, SPSS 20.0 statistical software was used to perform the statistical analysis of the data. The count data were expressed as \%. The data between groups are compared and analyzed with the $\chi^{2}$ test or the Fisher's exact test. The measurement data were expressed as mean \pm standard deviation (SD). The two groups of measurement data were compared using the $t$-test. $\mathrm{p}<0.05$ was considered statistically significant.

\section{RESULTS}

\section{The general data between the two groups} The general data between the two groups were shown in Table 1. After detailed comparison, the proportion of atrial fibrillation in the research group was lower than that in the conventional group, the difference was statistically significant $(\mathrm{p}<0.05)$. However, the remaining clinical indicators were not a significant difference between the two groups ( $\mathrm{p}>0.05$ ).

\section{Comparison of new-onset cerebral microbleeds in each group}

The new-onset CMB rates of the two groups are shown in Table 1. After surgery, the number of new-onset CMBs in the research group and the conventional group was 12 and 56 , accounting for 15.38 and $44.80 \%$ in each group, respectively. The research group had a much lower rate of new-onset CMBs than the conventional group $\left(\chi^{2}=18.656\right.$, $\mathrm{p}<0.001)$, and there was a significant difference in the data between the groups, and the difference was statistical significance.

\section{Comparison of National Institute of Health Stroke Scale, activity of daily living, and modified ranking scale scores between the two groups}

The NIHSS, ADL, and mRS scores of the two groups are shown in Figure 1. After surgery, the NIHSS of the research group and conventional group were significantly decreased with the increase of follow-up time. However, at each follow-up time, the NIHSS of the research group was much lower than that of the conventional group $(\mathrm{p}<0.05)$. The mRS scores of the two groups had a similar trend, while the ADL scores of the two groups had an opposite trend.

\section{Adverse reactions}

After comparison, the two groups of patients did not experience gum bleeding, massive bleeding, and blood transfusion. The difference between the groups was not significant ( $\mathrm{p}>0.05)$. 
Table 1. General data and new-onset CMB rate of the two groups $(n=203)$.

\begin{tabular}{|c|c|c|c|c|}
\hline & $\begin{array}{c}\text { Research Group } \\
(n=78)\end{array}$ & $\begin{array}{l}\text { Conventional } \\
\text { Group }(n=125)\end{array}$ & $\chi^{2} / t$ & p-value \\
\hline Age (years) & $70.45 \pm 8.13$ & $68.42 \pm 7.03$ & 1.883 & 0.061 \\
\hline Male/female (n) & $49 / 29$ & $85 / 40$ & 0.574 & 0.449 \\
\hline Smoking [n (\%)] & $35(44.87)$ & $60(48.00)$ & 0.189 & 0.664 \\
\hline Drinking [n (\%)] & $38(48.72)$ & $63(50.40)$ & 0.054 & 0.816 \\
\hline Atrial fibrillation [n (\%)] & $8(10.26)$ & $30(24.00)$ & 5.963 & $0.015^{*}$ \\
\hline Fasting blood glucose (mmol/L) & $5.73 \pm 1.26$ & $5.85 \pm 1.88$ & 0.498 & 0.619 \\
\hline Systolic blood pressure $(\mathrm{mmHg})$ & $142.17 \pm 16.35$ & $146.48 \pm 18.52$ & 1.686 & 0.093 \\
\hline Diastolic blood pressure $(\mathrm{mmHg})$ & $86.77 \pm 13.41$ & $88.45 \pm 10.10$ & 1.014 & 0.312 \\
\hline Total cholesterol (mmol/L) & $4.75 \pm 1.12$ & $4.96 \pm 1.04$ & 1.358 & 0.176 \\
\hline Low-density lipoprotein (mmol/L) & $3.03 \pm 0.94$ & $3.12 \pm 0.82$ & 0.719 & 0.473 \\
\hline Cause of stroke [n (\%)] & & & 3.197 & 0.362 \\
\hline Atherosclerotic stroke & $52(66.67)$ & $76(60.80)$ & & \\
\hline Cardiogenic cerebral embolism stroke & $12(15.38)$ & $32(25.60)$ & & \\
\hline Arteriolar occlusive stroke & $7(8.97)$ & $9(7.20)$ & & \\
\hline Other strokes with definite etiology & $7(8.97)$ & $8(6.40)$ & & \\
\hline New-onset CMB rate [n (\%)] & $12(15.38)$ & $56(44.80)$ & 18.656 & $<0.001 * *$ \\
\hline
\end{tabular}

CMBs: cerebral microbleeds. * $\mathrm{p}<0.05$ versus conventional group; ${ }^{* *} \mathrm{p}<0.001$ versus conventional group.

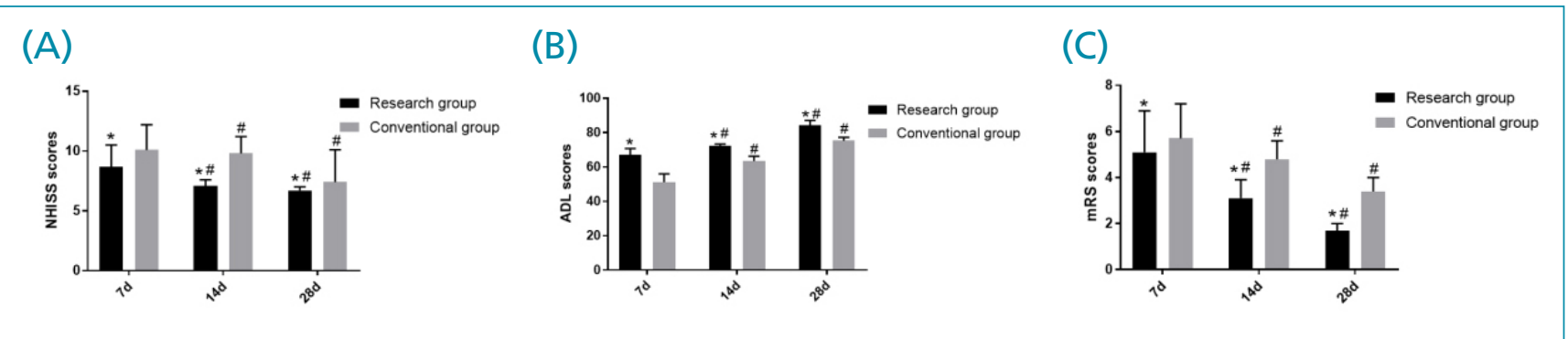

Figure 1. NIHSS: the National Institute of Health Stroke Scale; mRS: the modified ranking scale; ADL: activity of daily living. The NIHSS (A); ADL (B); and mRS (C) scores of the research group and conventional group after treatment. ${ }^{*} p<0.05$ versus conventional group; \#p<0.05 versus the score measured on day seven.

\section{DISCUSSION}

The emergence of new CMBs in the acute phase is a risk factor for symptomatic intracranial hemorrhage in patients with acute ischemic stroke treated with mechanical thrombectomy ${ }^{12-14}$. In the analysis of this study, the risk factors for CMBs are finally obtained, such as cardiogenic cerebral embolism, the use of antithrombotic drugs (especially anticoagulant drugs), etc. The relevant literature at the present stage has partially confirmed that there is no statistically significant difference between the data in the two cases of no hemorrhagic transformation and asymptomatic hemorrhage transformation in patients with mechanical thrombectomy for acute ischemic stroke. In addition, the research on the treatment of patients with asymptomatic hemorrhage transformation has been slow, and a more reliable improvement plan has not yet been obtained ${ }^{15}$. The proportion of patients with hemorrhagic transformation in the two groups participating in the study is basically the same as the relevant data in the previous literature. Finally, the conclusion on the effect of the application of tirofiban on the transformation of intracranial hemorrhage is more inclined to produce a favorable effect. However, it was found that the group of patients with acute ischemic stroke treated with tirofiban had significantly lower data of new-onset CMBs compared with the conventional group of patients treated with mechanical thrombectomy, indicating that the use of tirofiban could decrease the occurrence of CMBs after mechanical thrombectomy. 
Studies ${ }^{16}$ have shown that the risk factor for new CMBs is DWI-ASPECTS, ASPECTS is related to infarct size and NIH. The experimental data can only confirm that tirofiban has no significant effect on the increase of new-onset CMBs, but it cannot prove the clinical effect of the application of the drug in reducing new-onset CMBs. This result may be caused by the combined action of other influencing factors. The safety and feasibility of using tirofiban for the treatment of patients with acute ischemic stroke by mechanical thrombectomy are supported by various research data ${ }^{7-9}$. However, the clinical judgment of whether to use tirofiban is based on the patient's condition. The levels of antiplatelet strength, anticoagulation ratio, cardiogenic embolism, NIHSS, and other indicators in the two groups of patients with different treatment plans were compared and analyzed, and we found that the research group had a better patient report prognosis.

Antiplatelet therapy is one of the most commonly used regimens for mechanical thrombectomy in patients with acute ischemic stroke, and the combination of clopidogrel and aspirin has a very high clinical effect on patients with mild stroke with an early NIHSS of less than three, for example, it can significantly reduce the late recurrence rate of patients. In this study, the drug was administered according to the antiplatelet therapy guidelines. Compared with the conventional group, the proportion of patients in the study group who are treated with clopidogrel and aspirin is significantly higher, which suggests that the intensity of antiplatelet therapy in the study group is higher. In patients with platelet therapy, when the disease control effect is not good or even further development occurs, the clinical risk of using clopidogrel and aspirin combination therapy is lower. Tirofiban should be added to patients who have not been able to effectively control the development of the disease after the application of dual antiplatelet, so the antiplatelet strength is significantly higher in the study group compared to the conventional group. The application of anticoagulation therapy can significantly reduce the recurrence rate of patients with cardiogenic embolism, so most patients will use anticoagulation therapy. As a result, there is a certain difference in the data of anticoagulation and cardiogenic embolism between the two groups. The main reason is that the vast majority of patients using tirofiban had mild clinical symptoms at admission, so the NIHSS of the study group was significantly lower than that of the conventional group.

Antiplatelet drug treatment regimen occupies an important position in the clinical treatment of patients with clinical acute ischemic stroke, and the specific efficacy of intravenous antiplatelet drugs in patients with clinical acute ischemic stroke treated by mechanical thrombectomy is still in the stage of clinical exploration ${ }^{17}$. Research ${ }^{17}$ has confirmed that after mechanical thrombolytic blood flow is recanalized, maintenance for tirofiban is given to observe the efficacy of tirofiban. A total of 32 patients were enrolled this time, with $(16.1 \pm 4.4)$ as the average baseline NIHSS. After treatment, $75.15 \%$ of the patients showed recanalization, of which $43.75 \%$ was completely recanalized and $31.28 \%$ was partially recanalized. At the two-month follow-up, $56.26 \%$ of patients had an mRS score lower than three, indicating a good prognosis, only two patients had symptoms of symptomatic intracranial hemorrhage. The data of this study show that the effective treatment for patients with acute ischemic stroke is the use of tirofiban in the local arteries after the formation of mechanical thrombolytic antegrade blood flow in acute ischemic stroke. Arterial tirofiban can reduce the risk of reocclusion after intra-arterial thrombolysis.

Several limitations should be taken into consideration when interpreting the results of this analysis. The dosing regimens between the two groups were not completely consistent, especially the intra-arterial administration of tirofiban in the research group, which made this study not a well-controlled trial. The sample size of this study is still insufficient, and there is a lack of relatively rich literature reviews. Besides, the long-term efficacy and safety studies of tirofiban on stroke remain to be conducted.

\section{CONCLUSION}

In summary, clinical drug treatment for patients with ischemic stroke in the acute phase has certain safety and feasibility. Tirofiban treatment has the clinical effect of increasing the rate of vascular recanalization and reducing the risk of in-stent thrombosis for patients with acute ischemic stroke, safeguarding the safety of patients.

\section{AUTHORS' CONTRIBUTIONS}

YDS: Conceptualization, Data curation, Formal analysis, Writing - original draft, Writing - review \& editing. HJZ: Data curation. HL: Formal analysis. ZFJ: Formal analysis. LHW: Formal analysis. YYR: Formal analysis. XH: Formal analysis.

\section{REFERENCES}

1. Chen KN, Jiang W, Nie XM, Peng B, Wang N. Consensus of Chinese experts on postoperative monitoring and management of endovascular treatment for acute ischemic stroke. Chin Med J (Engl). 2019;67(56):78-345. https://doi.org/10.3760/ cma.j.issn.0376-2491.2017.03.002
2. Thorén M, Azevedo E, Dawson J, Egido JA, Falcou A, Ford GA, et al. Predictors for cerebral edema in acute ischemic stroke treated with intravenous thrombolysis. Stroke. 2017;48(9):246471. https://doi.org/10.1161/STROKEAHA.117.018223 
3. Sevick LK, Ghali S, Hill MD, Danthurebandara V, Lorenzetti $\mathrm{DL}$, Noseworthy $\mathrm{T}$, et al. Systematic review of the cost and cost-effectiveness of rapid endovascular therapy for acute ischemic stroke. Stroke. 2017;48(9):2519-26. https://doi. org/10.1161/STROKEAHA.117.017199

4. Yang M, Huo X, Miao Z, Wang Y. Platelet glycoprotein Ilb/lla receptor inhibitor tirofiban in acute ischemic stroke. Drugs. 2019;79(5):515-29. https://doi.org/10.1007/s40265-01901078-0

5. Gong J, Shang J, Yu H, Wan Q, Su D, Sun Z, et al. Tirofiban for acute ischemic stroke: systematic review and meta-analysis. Eur J Clin Pharmacol. 2020;76(4):475-81. https://doi.org/10.1007/ s00228-019-02817-8

6. Charidimou A, Karayiannis C, Song TJ, Orken DN, Thijs V, Lemmens $\mathrm{R}$, et al. Brain microbleeds, anticoagulation, and hemorrhage risk: meta-analysis in stroke patients with AF. Neurology. 2017;89(23):2317-26. https://doi.org/10.1212/ WNL.0000000000004704

7. Gao Q, Liu CM. Analysis of the efficacy and safety of tirofiban in mechanical thrombectomy for acute large vessel occlusion. Journal of Xuzhou Medical University. 2019;39(8):54. https:// doi.org/10.3969/j.issn.2096-3882.2019.08.001

8. Tan $X H$, Wang $H$, Zeng $Y Q$, li $X Q$, Du $H$. Effect of aspirin combined with clopidogrel and tirofiban in the treatment of elderly patients with progressive ischemic stroke. Clin Med. 2019;45(5):88-9. https://doi.org/10.19528/j.issn.10033548.2019.05.037

9. Cui FF, Jin AJ, Li YL, Shi QY, Li H. Efficacy of tirofiban combined with double antibody in the treatment of progressive stroke. Chinese Journal of Modern Medicine. 2019;29(16):69-74. https://doi.org/10.3969/j.issn. 1005-8982.2019.16.013

10. Niu WS, Liu Y, Wang YB, Ning Q, Li ZY. Effect of tirofiban on ischemic progressive stroke. Chinese Journal of Disease Control. 2019;5(4):65. https://doi.org/10.16462/j.cnki. zhjbkz.2019.04.024
11. Zhang LR. Comparison of efficacy and safety of tirofiban and double antibody in the treatment of progressive stroke. Medical Journal of Chinese People's Health. 2019 [cited on Dec. 6, 2019];31(14):7-9. Available from: http://lib.cqvip.com/Qikan/ Article/Detail?id=7100106083\&from=Qikan_Search_Index

12. Lu JJ, Zhang Y, Zahng QQ, Chen GF, Zheng W, Wang L, et al. Clinical study on 21 cases of acute cerebral infarction treated by micro catheter injection of tirofiban combined with solitaire stent thrombectomy. Shaanxi Medical Journal. 2019;48(6):740-3. https://doi.org/10.3969/j.issn.1000-7377.2019.06.016

13. Liu Q, Wang YL, Wang ZY, Peng ZQ, Xiang JF, Chen L. The application of intravenous and intra-arterial combined perfusion of tirofiban in treating acute ischemic stroke patients with no large vessel occlusion. J Intervent Rad. 2019 [cited on Dec. 12, 2019];28(12):1131-5. Available from: http://lib.cqvip.com/ Qikan/Article/Detail?id=7101005367\&from=Qikan_Search_ Index

14. Cui YX, Liang WS, Xu ZX. Application of tirofiban in the treatment of ischemic cerebrovascular disease. Journal of Apoplexy and Nervous Diseases. 2019 [cited on Jan. 3, 2019];36(1):92-4. Available from: https://d.wanfangdata.com. cn/periodical/ChlQZXJpb2RpY2FsQ0hJTmV3UzIwMjEwOTA 5EhJ6ZnlzampienoyMDE5MDEwMjYaCGI2enl1YjNj

15. Huang S, Zhou F, Zhang YD. Research progress of tirofiban in acute ischemic stroke. Chinese Journal of Cerebrovascular Disease. 2018;15(11):601-6. https://doi.org/10.3969/j. issn.1672-5921.2018.10.008

16. Niu JL, Ding $Y L$, Liu $Y$, Wei $C$, Zhang $Y$, Li L, et al. The influence of Tirofeban on new cerebral microhemorrhage in patients with acute ischemic stroke. Chinese Journal of Clinical Pharmacy. 2018;27(4):215-20. https://doi.org/10.19 577/j.1007-4406.2018.04.001

17. Dai Z, Liu XF. Advances in the treatment of acute ischemic stroke with Tirofeban. Chinese Journal of Cerebrovascular Disease. 2018;14(10):546-50. https://doi.org/10.3969/j. issn. 1672-5921.2017.10.010 